\title{
Prevalence of autoimmune diseases in in-patients with schizophrenia: nationwide population-based study $y^{\dagger}$
}

Shaw-Ji Chen, Yu-Lin Chao, Chuan-Yu Chen, Chia-Ming Chang, Erin Chia-Hsuan Wu, Chi-Shin Wu, Hsueh-Han Yeh, Chia-Hsiang Chen and Hui-Ju Tsai

\section{Background}

The association between autoimmune diseases and schizophrenia has rarely been systematically investigated.

\begin{abstract}
Aims
To investigate the association between schizophrenia and a variety of autoimmune diseases and to explore possible gender variation in any such association.

\section{Method}

Taiwan's National Health Insurance Research Database was used to identify 10811 hospital in-patients with schizophrenia and 108110 age-matched controls. Univariate and multiple logistic regression analyses were performed, separately, to evaluate the association between autoimmune diseases and schizophrenia. We applied the false discovery rate to correct for multiple testing.
\end{abstract}

\section{Results}

When compared with the control group, the in-patients with schizophrenia had an increased risk of Graves' disease (odds ratio $(\mathrm{OR})=1.32,95 \% \mathrm{Cl} 1.04-1.67)$, psoriasis $(\mathrm{OR}=1.48$, $95 \% \mathrm{Cl} 1.07-2.04)$, pernicious anaemia $(\mathrm{OR}=1.71,95 \% \mathrm{Cl}$ 1.04-2.80), celiac disease $(\mathrm{OR}=2.43,95 \% \mathrm{Cl} 1.12-5.27)$ and hypersensitivity vasculitis $(\mathrm{OR}=5.00,95 \% \mathrm{Cl} 1.64-15.26)$, whereas a reverse association with rheumatoid arthritis $(\mathrm{OR}=0.52,95 \% \mathrm{Cl} 0.35-0.76)$ was also observed. Genderspecific variation was found for Sjögren syndrome, hereditary haemolytic anaemia, myasthenia gravis, polymyalgia rheumatica and dermatomyositis.

\section{Conclusions}

schizophrenia was associated with a greater variety of autoimmune diseases than was anticipated. Further investigation is needed to gain a better understanding of the aetiology of schizophrenia and autoimmune diseases.

\section{Declaration of interest}

None.
Schizophrenia is a devastating neuropsychiatric illness and a complex disease, and both genetic components and environmental factors play a role in its development. ${ }^{1-3}$ In general, around $1 \%$ of the total population is affected by schizophrenia. ${ }^{4}$ However, despite a considerable number of studies, the aetiology of schizophrenia remains unclear. In addition, people with schizophrenia often develop somatic symptoms, particularly autoimmune disorders. ${ }^{4}$ Previous studies have reported that, compared with the general population, individuals with schizophrenia have a different prevalence of some autoimmune diseases and experience different immunological responses, ${ }^{4,5}$ which suggests that aberrant autoimmune responses may play a role in the aetiology of schizophrenia. Autoimmune involvement may account for the high rate of perinatal obstetric complications observed in people with schizophrenia and may have potential influence on central nervous system infection, which generates antibodies against the brain tissue in those with schizophrenia. ${ }^{6,7}$ In addition, a number of genetic studies have found that human leukocyte antigens were associated with schizophrenia; however, the results were inconsistent across different studies. ${ }^{8-10}$ Therefore, investigating the association between autoimmune diseases and schizophrenia is particularly important to facilitate a dissection of the aetiology of schizophrenia.

To our knowledge, to date only Eaton et al has simultaneously examined a number of autoimmune diseases in relation to schizophrenia in a Danish population. ${ }^{11}$ They used data from the Danish national registries from 1981 to 1988 to evaluate the association between schizophrenia and 29 autoimmune diseases. They found that a positive association existed between

†See editorial, pp. 353-355, this issue. schizophrenia and a variety of autoimmune diseases, ${ }^{11}$ and concluded that schizophrenia was associated with a larger variety of autoimmune diseases than was expected. Since then, no study has applied a similar approach to examine the association between autoimmune diseases and schizophrenia, mainly due to the limited sample size in a single study. Few efforts have been directed to study this connection in Asian populations; only Chang et al has reported a connection between autoimmune diseases and schizophrenia. ${ }^{12}$ In particular, they measured the messenger ribonucleic acid expression levels of a number of cytokines in the monocytes of people with schizophrenia. Although their findings demonstrated a suggestive increased autoimmunological response in participants with schizophrenia, the association between schizophrenia and autoimmune diseases has not yet been systematically investigated. To address this issue, we turned to the 2005 National Health Insurance data in Taiwan. First, we compared the prevalence of 34 autoimmune diseases in in-patients with schizophrenia with that of a control group of individuals without schizophrenia from the general population. Next, we examined the association between autoimmune diseases and schizophrenia, and further explored possible gender variation in such connections.

\section{Method}

\section{Study population}

The data used in this study were obtained from Taiwan's National Health Insurance Research Database (NHIRD) for 2005. The NHIRD is derived from the National Health Insurance Program (NHIP), which was launched in 1995. In detail, the NHIP 
provides mandatory comprehensive medical care services to residents of Taiwan. Since 1995, the NHIRD has collected the data of all ambulatory care and in-patient claims from NHIP enrolees, which represent over $98 \%$ of the total population in Taiwan. ${ }^{13}$ Therefore, the NHIRD provides a valuable data source and a unique opportunity to undertake the objectives addressed in this study.

In-patients with schizophrenia were identified from the Psychiatric Inpatient Medical Claim Dataset (PIMCD) of the NHIRD from 2005. Specifically, participants with a primary diagnosis of schizophrenia (ICD-9-CM ${ }^{14}$ code: 295$)^{15}$ and having at least one claim for using the in-patient service for schizophrenia treatment in 2005 were included in the study. A total of 10811 in-patients with schizophrenia (schizophrenia group) were identified in this data-set.

The comparison participants (control group) were identified from the Longitudinal Health Insurance Database 2005 (LHID2005) of the NHIRD; they had no reports of any diagnosis of schizophrenia in 2005. In detail, the LHID2005 contains all the registration and claim data for 1000000 individuals, which were collected by the NHIP and randomly sampled from the 2005 Registry for Beneficiaries of the NHIRD. Detailed information on the sampling method representative of the LHID2005 is provided at: http://w3.nhri.org. tw/nhird/date cohort.htm\#1. In addition, participants in the control group were matched with participants in the schizophrenia group by age group (by 10-year interval) with a ratio of 1:10 and were randomly drawn from the LIHD2005. A total of 108110 age-matched comparison participants were identified in this data-set.

\section{Definition of autoimmune diseases}

We determined the autoimmune diseases to be included in our analysis on the basis of 'the list of catastrophic illnesses of rheumatology' and those with autoimmune properties provided by the NHIP. Of note, this list represents the most serious autoimmune diseases with a heavy economic burden in Taiwan. As a result, a total of 34 autoimmune diseases were considered and identified based on their ICD-9-CM codes (see Appendix). For both the schizophrenia and control groups, medical records for any of the 34 autoimmune diseases were retrieved from the in-patient and ambulatory services in the NHIRD in 2005.

\section{Statistical analyses}

We first computed and compared the distribution of demographic characteristics between the schizophrenia group and the control group using the $\chi^{2}$ test, including age $(<29,30-39,40-49,50-$ 59 and 60 years); gender; region (northern, central, southern and eastern); urbanicity (urban, suburban and rural); enrolee category (a proxy measure of socioeconomic status); and information regarding health system utilisation such as number of hospital admissions in 2005 and length of hospital stay in 2005. Detailed information regarding enrolee category has been provided elsewhere. ${ }^{16}$ Briefly, the insurance fee was determined based on enrolees' wages as indicated in the NHIP. Participants were put into four enrolee categories, with wages of those in the first group (I) higher than those in subsequent categories (II-IV).

Next, we computed the prevalence rates for all examined autoimmune diseases among both the schizophrenia and control groups. Univariate and multiple logistic regression analyses were performed, separately, to evaluate the association between selected autoimmune diseases and schizophrenia. The covariates adjusted in the multiple conditional logistic regression models were gender, enrolee category and region. In addition, to examine the gender effect between autoimmune diseases and schizophrenia, gender-stratified analysis was carried out, which included the covariates enrolee category and region. All of the analyses were performed using SAS version 8.2 for Windows. Furthermore, to control for potential false positive results due to multiple testing, we applied the false discovery rate (FDR) to correct for multiple testing. ${ }^{17}$ The FDR procedure was performed using statistical packages R 2.10.0 (www.r-project.org/) and Bioconductor 2.7 (www.bioconductor.org/), both run on Windows.

\section{Results}

\section{Study sample characteristics}

A total of 118921 participants (10811 in the schizophrenia group and 108110 in the control group) were included in this study. Table 1 shows the distribution of demographic characteristics in both groups. In general, the distribution of gender, enrolee categories (a proxy measure of socioeconomic status), region, urbanicity, average number of hospital admissions in 2005 and length of hospital stay in 2005 are significantly different between the schizophrenia group and the control group. Compared with the control group there were a higher percentage of males among the schizophrenia group $(54.9 \%$ v. $49.5 \%)$, a higher number of hospital admissions in 2005 (1.15 v. 0.10 times) and a longer hospital stay in 2005 ( $28.38 v .0 .44$ days). The percentage of those in enrolee category IV was greater in the schizophrenia group than that in the control group $(57.2 \%$ v. $15.4 \%)$. In other words, those in the schizophrenia group tended to have a lower socioeconomic

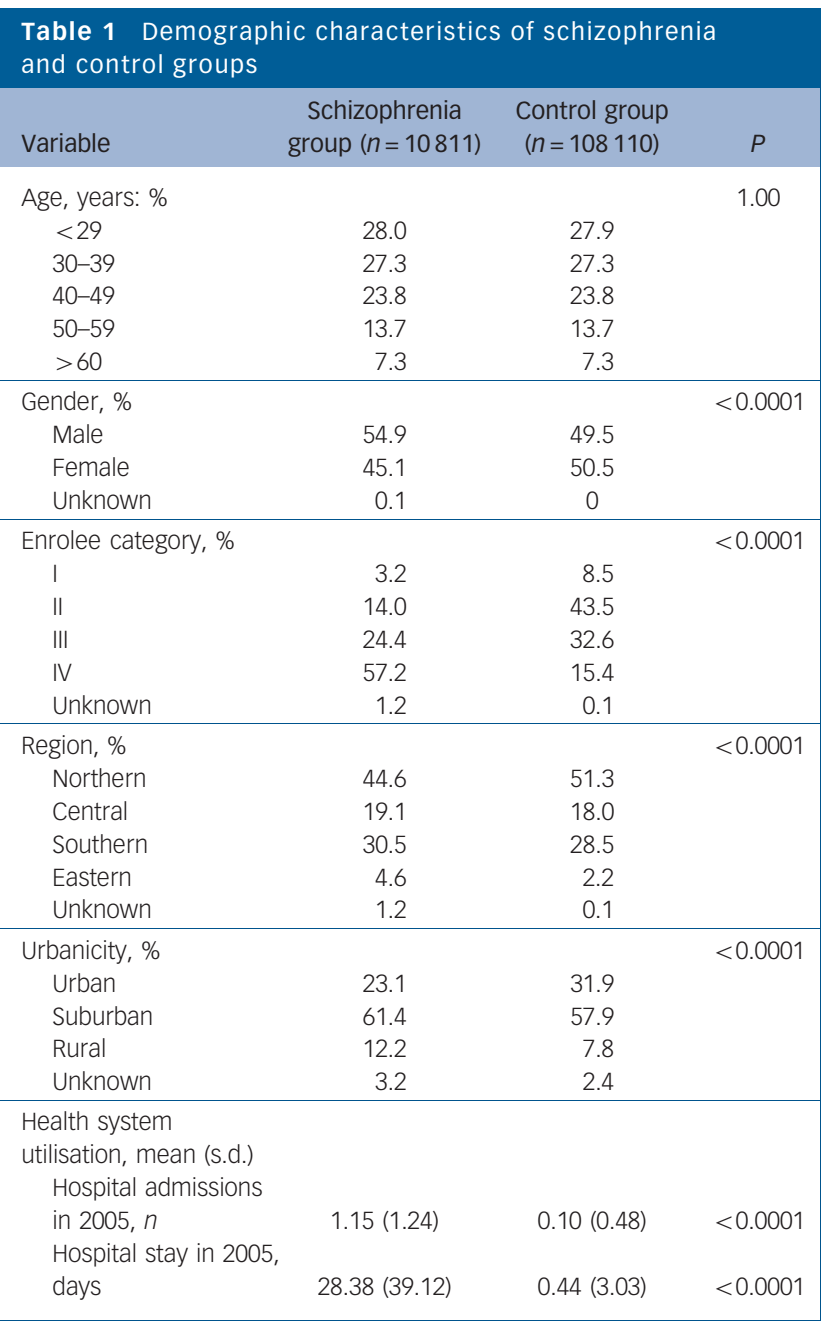




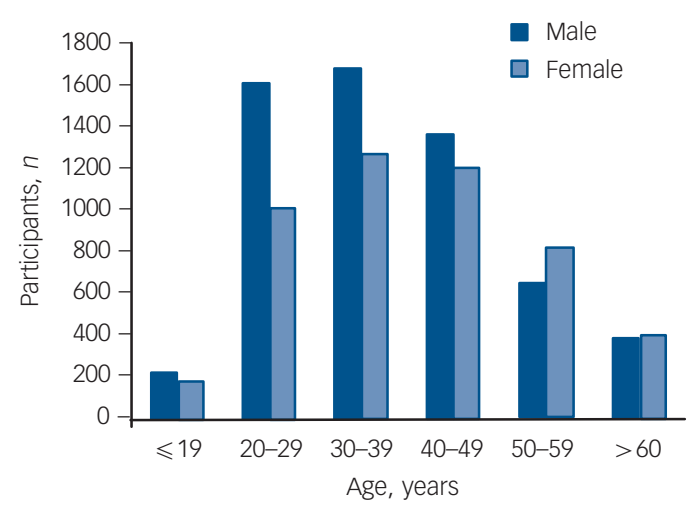

Fig. 1 Number of in-patients with schizophrenia across different age groups, stratified by gender.

status than those in the control group. Additionally, when we grouped the study sample by age, we found that the highest number of participants in the schizophrenia group were aged 30-39 years for both males and females (Fig. 1).

\section{Prevalence of autoimmune diseases}

Next, we calculated and compared the annual prevalence of 34 autoimmune diseases among both groups in 2005 (Table 2 and online Table DS1). In the schizophrenia group, 14 out of 34 autoimmune diseases had a higher prevalence than that found in the control group (Table 2 and online Table DS1). When stratifying by gender, in the schizophrenia group the prevalence of 16 autoimmune diseases was higher in females than in males. Conversely, among the control group the prevalence of 21 autoimmune diseases was higher in females than in males (Table 2 and online Table DS1).

\section{Association of autoimmune diseases with schizophrenia}

For the 16 autoimmune diseases where the number of individuals with these conditions was $\geqslant 5$ in the schizophrenia group, we further examined the association between autoimmune diseases and schizophrenia. In particular, when compared with the comparison group, participants in the schizophrenia group had a significantly higher risk of Graves' disease (odds ratio $(\mathrm{OR})=1.32$, 95\% CI 1.04-1.67), psoriasis $(\mathrm{OR}=1.48,95 \% \mathrm{CI}$ 1.07-2.04), pernicious anaemia $(\mathrm{OR}=1.71,95 \%$ CI 1.04-2.80), celiac disease $(\mathrm{OR}=2.43,95 \% \mathrm{CI} 1.12-5.27)$ and hypersensitivity vasculitis $(\mathrm{OR}=5.00,95 \%$ CI $1.64-15.26)$; whereas a reverse association with rheumatoid arthritis $(\mathrm{OR}=0.52$, 95\% CI 0.35 0.76) was observed (Table 3). Of note, the positive association between hypersensitivity vasculitis and schizophrenia remained significant after correcting for multiple testing. Likewise, the reverse association of rheumatoid arthritis with schizophrenia also remained significant after accounting for multiple corrections.

\section{Gender effect of autoimmune diseases on schizophrenia}

In addition, we performed a gender-stratified analysis to examine the gender effect (Table 3). We observed a gender-specific effect for Sjögren syndrome, hereditary haemolytic anaemia, myasthenia gravis, polymyalgia rheumatica and dermatomyositis, although none of these observed associations were statistically significant (Table 3).

Furthermore, Graves' disease, psoriasis and dermatomyositis were significantly associated only with males in the schizophrenia group. Whereas, type 1 diabetes, hereditary haemolytic anaemia, celiac disease and hypersensitivity vasculitis were significantly associated only with females in the schizophrenia group, and in addition there was a significant inverse association of rheumatoid arthritis with females in this group. Among those autoimmune diseases found to be significantly associated with the schizophrenia group, only Graves' disease in males and rheumatoid arthritis in

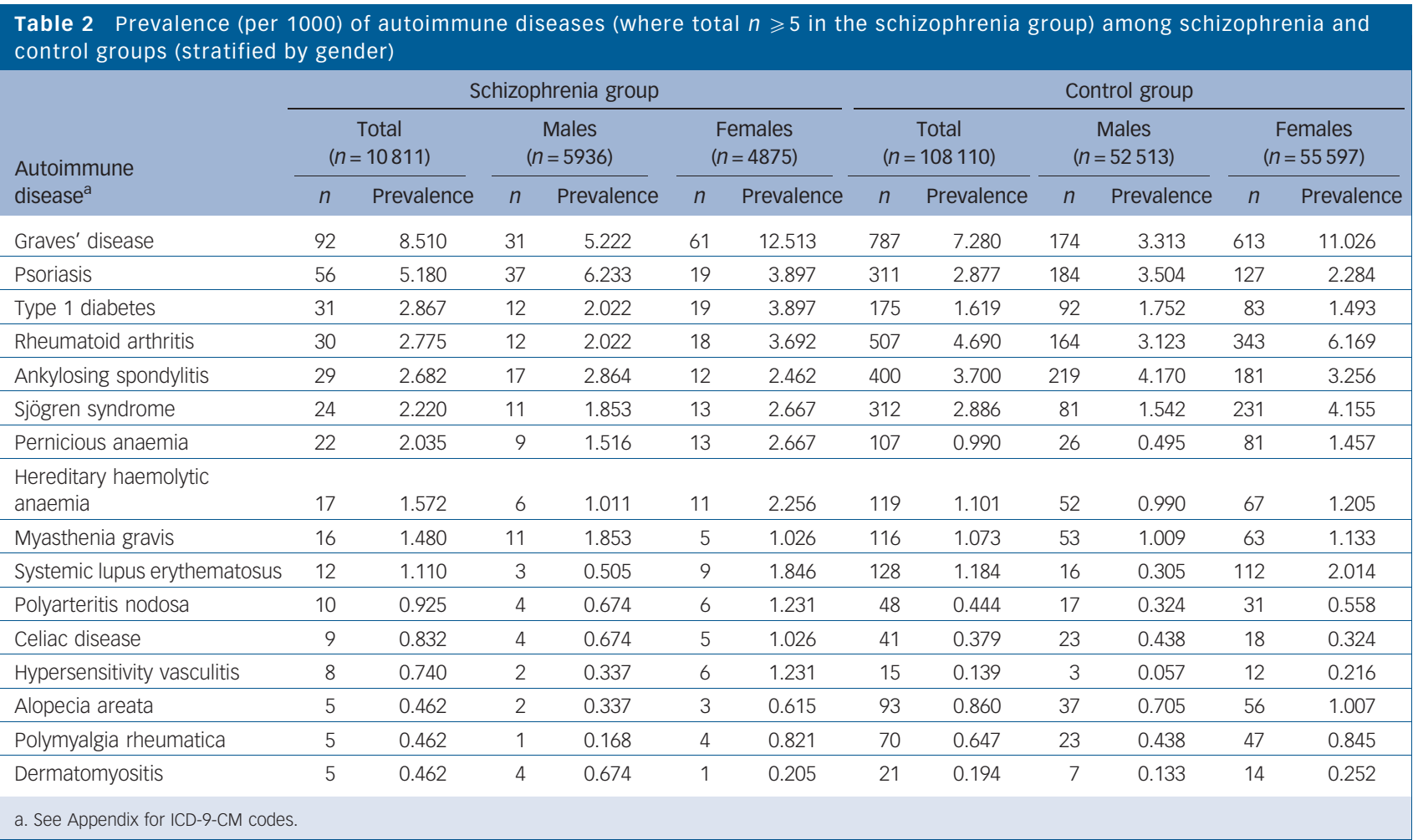




\begin{tabular}{|c|c|c|c|c|c|}
\hline \multirow[b]{2}{*}{ Autoimmune diseases ${ }^{a}$} & \multirow{2}{*}{$\frac{\text { Total }}{\text { Adjusted } \mathrm{OR}^{\mathrm{b}}(95 \% \mathrm{Cl})}$} & \multicolumn{2}{|c|}{ Males } & \multicolumn{2}{|c|}{ Female } \\
\hline & & Crude OR $(95 \% \mathrm{Cl})$ & Adjusted $\mathrm{OR}^{\mathrm{C}}(95 \% \mathrm{Cl})$ & Crude OR $(95 \% \mathrm{Cl})$ & Adjusted $\mathrm{OR}^{\mathrm{C}}(95 \% \mathrm{Cl})$ \\
\hline Graves' disease & $1.32^{\mathrm{d}}(1.04-1.67)$ & $1.5 \mathbf{9}^{\mathrm{d}}(1.08-2.34)$ & $1.86^{\mathrm{e}}(1.22-2.84)$ & $1.12(0.86-1.47)$ & $1.16(0.87-1.54)$ \\
\hline Psoriasis & $1.48^{\mathrm{d}}(1.07-2.04)$ & $\mathbf{1 . 8 1}^{\mathrm{e}}(1.26-2.59)$ & $1.60^{\mathrm{d}}(1.08-2.36)$ & $1.15(0.86-2.44)$ & $1.26(0.70-2.26)$ \\
\hline Type 1 diabetes & $1.54(0.99-2.37)$ & $1.46(0.80-2.70)$ & $1.18(0.62-2.25)$ & $2.39^{\mathrm{e}}(1.39-4.11)$ & $1.98^{\mathrm{d}}(1.11-3.54)$ \\
\hline Rheumatoid arthritis & 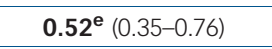 & $0.66(0.37-1.19)$ & $0.59(0.32-1.08)$ & $\mathbf{0 . 5 9 ^ { d }}(0.36-0.94)$ & $\mathbf{0 . 4 8}^{\mathrm{e}}(0.30-0.79)$ \\
\hline Ankylosing spondylitis & $0.71(0.47-1.06)$ & $0.70(0.43-1.15)$ & $0.71(0.42-1.21)$ & $0.74(0.41-1.33)$ & $0.71(0.38-1.34)$ \\
\hline Sjögren syndrome & $0.92(0.59-1.41)$ & $1.23(0.65-2.30)$ & $1.30(0.82-3.14)$ & $0.63(0.36-1.10)$ & $0.68(0.38-1.20)$ \\
\hline Pernicious anaemia & $1.71^{\mathrm{d}}(1.04-2.80)$ & $3.13^{\mathrm{e}}(1.46-6.67)$ & $1.91(0.84-4.35)$ & $1.80^{d}(1.00-3.23)$ & $1.57(0.84-2.93)$ \\
\hline Hereditary haemolytic anaemia & $1.45(0.84-2.50)$ & $1.04(0.45-2.43)$ & $0.95(0.39-2.35)$ & $1.84(0.97-3.48)$ & $1.99^{\mathrm{d}}(1.00-3.93)$ \\
\hline Myasthenia gravis & $1.18(0.67-2.09)$ & $1.87(0.98-3.59)$ & $1.42(0.69-2.92)$ & $0.89(0.36-2.21)$ & $0.86(0.34-2.23)$ \\
\hline Systemic lupus erythematosus & $1.22(0.66-2.27)$ & $1.69(0.49-5.81)$ & $2.78(0.76-10.12)$ & $0.90(0.46-1.78)$ & $1.02(0.50-2.07)$ \\
\hline Polyarteritis nodosa & $2.04(0.95-4.39)$ & $2.12(0.72-6.31)$ & $1.91(0.50-7.22)$ & $2.17(0.90-5.20)$ & $2.19(0.86-5.58)$ \\
\hline Celiac disease & $2.43^{\mathrm{d}}(1.12-5.27)$ & $1.57(0.54-4.54)$ & $1.51(0.49-4.66)$ & $3.12^{\mathrm{d}}(1.16-8.39)$ & $4.10^{d}(1.42-11.78)$ \\
\hline Hypersensitivity vasculitis & $5.00^{\mathrm{e}}(1.64-15.26)$ & $4.51(0.41-49.75)$ & $5.65(0.51-62.41)$ & $4.48^{d}(1.41-14.29)$ & $4.69^{d}(1.34-16.45)$ \\
\hline Alopecia areata & $0.72(0.29-1.83)$ & $0.49(0.12-2.02)$ & $0.65(0.15-2.82)$ & $0.60(0.19-1.92)$ & $0.78(0.24-2.57)$ \\
\hline Polymyalgia rheumatica & $0.77(0.30-1.98)$ & $0.39(0.05-2.90)$ & $0.39(0.05-3.03)$ & $0.95(0.34-2.65)$ & $1.02(0.35-2.95)$ \\
\hline Dermatomyositis & $2.55(0.88-7.39)$ & $\mathbf{5 . 1 6 ^ { \mathrm { d } } ( 1 . 5 1 - 1 7 . 6 2 )}$ & $\mathbf{5 . 1 1 ^ { \mathrm { d } } ( 1 . 2 5 - 2 0 . 9 3 )}$ & $0.80(0.11-6.08)$ & $0.93(0.11-7.62)$ \\
\hline
\end{tabular}

females remained statistically significant after correction for multiple testing (Table 3).

\section{Discussion}

In general, schizophrenia and autoimmune diseases share many common clinical features. First, the diagnostic procedures are largely dependent on clinical criteria such as lacking a specific aetiological diagnosis. Second, the treatments are usually palliative, not curative. Third, schizophrenia and most autoimmune diseases have unequal distributions of incidence for age, gender, cultures and across different ethnic groups. ${ }^{18,19}$ However, schizophrenia is more prevalent among males, whereas most of the autoimmune diseases disproportionally affect females. Finally, both are complex disorders; that is, disorders that are caused by multiple genetic, environmental and lifestyle factors and the interaction between these factors, and they do not follow a clear Mendelian pattern of inheritance. Furthermore, due to limited knowledge of pathophysiology, individuals with schizophrenia and/or certain autoimmune diseases might be similar in clinical presentation, but their underlying aetiologies are very heterogeneous. Therefore, using large medical databases to identify whether certain autoimmune diseases co-occur with schizophrenia more or less commonly than expected by chance should provide an insight into the possible shared genetic, immunological and environmental mechanisms of both disorders. To our knowledge, this is the first study using a national database to address the association between schizophrenia and autoimmune diseases in an Asian population. More importantly, our data indicated that a certain number of autoimmune diseases were significantly associated (either positively or negatively) with in-patients with schizophrenia.

\section{Significant association between autoimmune diseases and schizophrenia}

Overall, a significant increased risk for Graves' disease, psoriasis, pernicious anaemia, celiac disease and hypersensitivity vasculitis were found in the schizophrenia group, whereas a significant reverse association was observed between rheumatoid arthritis and this group. Several studies have reported the association of Graves' disease and rheumatoid arthritis with schizophrenia, respectively, which we discuss below.

Graves' disease, also known as thyrotoxicosis, is an autoimmune disease that affects up to $2 \%$ of the female population, and has a female-to-male incidence ratio ranging from 5:1 to $10: 1 .^{20,21}$ In agreement with previous studies, ${ }^{1,22,23}$ our present study revealed that participants with schizophrenia had a higher prevalence of Graves' disease than did those in the comparison group. In addition, thyroid hormone dysfunction per se can result in cognitive function impairment, and emotional and behavioural disturbance. Therefore, further investigation of the autoimmune process of Graves' disease in individuals with schizophrenia is warranted.

The inverse relationship between rheumatoid arthritis and schizophrenia observed in our study is generally consistent with previous findings in the area of autoimmune diseases. ${ }^{3,24-26}$ It has been proposed that this relationship may be attributed to the interplay of genetic influence (hitch-hiking of major histocompatibility complex genes), environmental factors (institutionalisation and sedentary lifestyle due to negative symptoms), immunological aspects (common infectious aetiology and immunity) and pharmacological effects (anti-inflammatory and analgesic effects of antipsychotics). ${ }^{27}$ Specifically, rheumatoid arthritis is a progressive, systemic inflammatory disorder that may affect many tissues and organs, but mainly attacks synovial joints. The peak onset of rheumatoid arthritis occurs between the fourth to sixth decade of life, and women are two to five times more likely than men to develop this disease. ${ }^{28}$ Of interest, as shown in Table 3, we observed a significant inverse association of rheumatoid arthritis with females in the schizophrenia group. It will be of importance in future studies to dissect the relationship between rheumatoid arthritis and schizophrenia to gain a better understanding of the interplay of the four components listed above. 


\begin{tabular}{|c|c|c|c|c|}
\hline Autoimmune disease & $\begin{array}{l}\text { Country of study } \\
\text { population }\end{array}$ & $\begin{array}{l}\text { Prevalence in general } \\
\text { population in our study }\end{array}$ & $\begin{array}{l}\text { Prevalence in the } \\
\text { compared study }\end{array}$ & Source \\
\hline Graves' disease & China & 7.3 & 12 & Shapira et $a l^{29}$ \\
\hline Rheumatoid arthritis & China & 4.7 & $2-9.3$ & Zeng et a/ ${ }^{30}$ \\
\hline Psoriasis & Japan & 2.9 & $2.4-11.8$ & Raychaudhuri et al ${ }^{\beta 1}$ \\
\hline Pernicious anaemia & China & 1.0 & 1.3 & Chan $e t a l^{32}$ \\
\hline Celiac disease & India & 0.4 & $0.2-2$ & Cummins \& Roberts-Thomson ${ }^{33}$ \\
\hline Hypersensitivity vasculitis & India & 0.1 & $0.03-0.1$ & Watts $^{34}$ \\
\hline
\end{tabular}

\section{Comparison of autoimmune disease prevalence found in the present study and Asian populations}

We compared the prevalence of the 34 autoimmune diseases we had investigated in the general population in Taiwan to that reported in Asian populations. The data in Table 4 show the comparisons between the prevalence of the six significant autoimmune diseases observed in Table 3 and the prevalence of these reported in Asian populations. We found that the prevalence of these six conditions was comparable with that reported by other groups (Table 4).

\section{Genetic influences on schizophrenia and autoimmune diseases}

Recent genome-wide association studies have identified a growing number of disease-associated loci and provided enormous insight into the aetiology of complex genetic disorders, including schizophrenia and autoimmune diseases. For example, using molecular pathway-based analysis, genome-wide association studies of schizophrenia revealed a number of genes involved in neuronal cell adhesion, membrane scaffolding, apoptosis, glutamate metabolism or synaptogenesis. ${ }^{35,36}$ Meanwhile, genome-wide association studies of autoimmune diseases have highlighted immune-associated genes involved in lymphocyte activation (receptor signalling and co-stimulation pathways), microbial recognition and cytokines or cytokine receptors. ${ }^{37}$ Strikingly, consistent with the results from previous linkage and case-control association studies, genome-wide association study results confirmed a shared genetic association in the major histocompatibility complex (also known as human leukocyte antigen) region between schizophrenia and most autoimmune diseases. ${ }^{38,39}$ The unique coding and non-coding genetic variation of human leukocyte antigen alleles are involved in shaping the nature of antigen responses to self- or non-self-antigens. Moreover, extensive allelic variation and linkage disequilibrium (non-random or correlated association of alleles due to physical closeness) have been observed throughout the major histocompatibility complex region. ${ }^{40}$ Therefore, this shared gene region of association may influence direct involvement of certain human leukocyte antigens, or it may be through linkage disequilibrium between the loci related to schizophrenia and those related to autoimmune diseases in human leukocyte antigen regions. Genotyping more dense single-nucleotide polymorphisms or deep sequencing human leukocyte antigen regions may shed more light on the nature of the common shared genetic components of schizophrenia and autoimmune diseases.

\section{Strengths and limitations}

This study has several strengths. First, the NHIP in Taiwan is a single-payer compulsory social insurance plan that centralises the disbursement of healthcare funds. The system promises equal access to healthcare for all citizens, and it covers nearly all clinical activities in Taiwan. More importantly, as mentioned earlier, the NHIP covers over $98 \%$ of the total population in Taiwan. Second, unlike several national registry databases that exist in European countries such as Iceland, Finland and Denmark, NHIRD in Taiwan is the only national database that exists among Asian countries. Third, the sample size in this study is sufficient since it is based on the NHIRD data (i.e. on national data). Since autoimmune diseases and schizophrenia are both considered rare diseases, a large sample size makes it feasible to investigate the relationship between them.

However, some limitations should also be considered. First, the NHIRD did not collect laboratory data to validate the physician diagnosis or the severity of autoimmune diseases. However, most autoimmune diseases examined in this study were selected based on the list of catastrophic illness of rheumatology provided by the NHIP. Based on standard clinical guidance for the list of catastrophic illnesses in the NHIP, it requires three board-certified rheumatologists to independently diagnose individuals with the explicit ICD-9-CM code for the autoimmune diseases on the list of catastrophic illnesses. As such, the diagnosis of autoimmune diseases was relatively reliable. Second, due to the nature of the cross-sectional design, this study cannot investigate temporal/causal relationships between autoimmune disorders and schizophrenia, but can only document the prevalence and association between schizophrenia and autoimmune diseases. Indeed, the observed excess in autoimmune diseases associated with schizophrenia may be partly explained by non-aetiological causational mechanisms. For example, because of the long course of treatment, people with schizophrenia were more likely to be treated as compared with individuals in the community without schizophrenia. It will be of importance to further investigate the causal relationship between schizophrenia and autoimmune diseases using a prospective cohort. Finally, this is the first study of its kind to be conducted in an Asian population. Therefore, the results observed in this study may or may not be generalised to other ethnic groups, and caution is needed when interpreting the results of this study.

\section{Further research}

In the present study, we documented that in an Asian population schizophrenia was associated with a wider variety of autoimmune diseases than was expected. The findings of comorbidity suggest that shared genetic components and shared aetiological pathways may occur in autoimmune diseases and schizophrenia. Further investigation of the interplay between genetic, immune and pathological mechanisms will provide a better understanding of the underlying aetiology of both schizophrenia and autoimmune diseases. 
Shaw-Ji Chen, MD, Yuli Mental Research Center, Department of Psychiatry, Yuli Veteran Hospital, Hualien, and Institute of Medical Science, Tzu Chi University, Hualien, Taiwan; Yu-Lin Chao, MD, Institute of Medical Science, Tzu Chi University, Hualien, Taiwan; Chuan-Yu Chen, PhD, Institute of Public Health, School of Medicine, National Yang-Ming University, Taipei, and Division of Biostatistics and Bioinformatics, Institute of Population Health Sciences, National Health Research Institutes, Miaoli, Taiwan; Chia-Ming Chang, MD, PhD, Department of Psychiatry, Chang Gung Memorial Hospital, Lin-Kou \& Chang Gung University, Taoyuan, Taiwan; Erin Chia-Hsuan $\mathbf{W u}, \mathrm{MD}$, Graduate Institute of Humanity in Medicine, Taipei Medical University, Taipei, Taiwan; Chi-Shin Wu, MD, Department of Psychiatry, Far Eastern Memorial Hospital, Taipei, Taiwan; Hsueh-Han Yeh, MS, Chia-Hsiang Chen MD, PhD, Division of Mental Health and Addiction Medicine, Institute of Population Health Sciences, National Health Research Institutes, Miaoli, Taiwan; Hui-Ju Tsai, MPH, PhD, Department of Pediatrics, Feinberg School of Medicine, Northwestern University, Chicago, Illinois, USA, and Division of Biostatistics and Bioinformatics, Institute of Population Health Sciences, National Health Research Institutes, Miaoli, Taiwan

Correspondence: Hui-Ju Tsai, MPH, PhD, Division of Biostatistics and Bioinformatics, Institute of Population Health Sciences, National Health Research Institutes, 35 Keyan Road, Zhunan Town, Miaoli County 350, Taiwan. Email: Institutes, 35 Keyan

First received 18 Jan 2011, final revision 17 Oct 2011, accepted 11 Nov 2011

\section{Funding}

This study was supported in part by grants from the National Health Research Institutes (PH-099-PP-56, PH-100-PP-14).

\section{Acknowledgements}

Our thank to Drs Kuan-Yi Wu and Hsin-Yi Liang for their comments on the manuscript Lan-Chao Wang for his assistance with data management and Tami R. Bartell for English editing. This study is based in part on data from the National Health Insurance Research Database provided by the Bureau of National Health Insurance, Department of Health and managed by the National Health Research Institutes of Taiwan.

\section{Appendix}

\section{Autoimmune disease included in analysis with ICD-9-CM codes}

Graves' disease $(242,242.01)$, Crohn's disease (555.0, 555.1, 555.2, 555.9), psoriasis (696, 696.1, 696.8), systemic lupus erythematosus (710.0), rheumatoid arthritis (714), ankylosing spondylitis (720), Guillain-Barré syndrome (357.0), type 1 diabetes (250.01, 250.03, 250.11, 250.13, $250.21,250.23,250.31,250.33,250.41,250.43,250.51,250.53,250.61$ $250.63,250.71,250.73,250.81,250.83,250.91,250.93)$, Sjögren syndrome (710.2), myasthenia gravis (358), pernicious anaemia (281), hereditary haemolytic anaemia (282), polyarteritis nodosa (446), celiac disease (579), uveitis $(364.00,364.01)$, polymyalgia rheumatica (725), dermatomyositis (710.3), Hashimoto's thyroiditis (245.2), hypersensitivity vasculitis (446.2, 446.29), Behcet's disease (136.1), polymyositis (710.4), alopecia areata (704.01), Wegener's granulomatosis (446.4), ulcerative colitis (556.0, 556.6, 556.8, 556.9), autoimmune haemolytic anaemia (283), pemphigus (694.4), multiple sclerosis (340), systemic sclerosis (710.1), juvenile rheumatoid arthritis $(714.30,714.33)$, Goodpasture syndrome (446.21), giant cell arteritis (446.5), thromboangitis obliterans (443.1), arteritis obliterans (446.7) and Kawasaki disease (446.1).

\section{References}

1 van OS J, Kapur S. Schizophrenia. Lancet 2009; 374: 635-45.

2 O'Donovan MC, Craddock NJ, Owen MJ. Genetics of psychosis: insights from views across the genome. Hum Genet 2009; 126: 3-12.

3 Leucht S, Burkard T, Henderson J, Maj M, Sartorius N. Physical illness and schizophrenia: a review of the literature. Acta Psychiatr Scand 2007; 116: 317-33

4 Jones AL, Mowry BJ, Pender MP, Greer JM. Immune dysregulation and self-reactivity in schizophrenia: do some cases of schizophrenia have an autoimmune basis? Immunol Cell Biol 2005; 83: 9-17.

5 Strous RD, Shoenfeld Y. Schizophrenia, autoimmunity and immune system dysregulation: a comprehensive model updated and revisited. $J$ Autoimmun 2006; 27: 71-80
6 Kirch DG. Infection and autoimmunity as etiologic factors in schizophrenia: a review and reappraisal. Schizophr Bull 1993; 19: 355-70.

7 Chengappa KN, Nimgaonkar VL, Bachert C, Yang ZW, Rabin BS, Ganguli R. Obstetric complications and autoantibodies in schizophrenia. Acta Psychiatr Scand 1995; 92: 270-3.

8 Li T, Underhill J, Liu XH, Sham PC, Donaldson P, Murray RM, et al. Transmission disequilibrium analysis of HLA class II DRB1, DQA1, DQB1 and DPB1 polymorphisms in schizophrenia using family trios from a Han Chinese population. Schizophr Res 2001; 49: 73-8.

9 Schwab SG, Hallmayer J, Lerer B, Albus M, Borrmann M, Honig S, et al. Support for a chromosome 18p locus conferring susceptibility to functional psychoses in families with schizophrenia, by association and linkage analysis Am J Hum Genet 1998; 63: 1139-52.

10 Palmer CG, Hsieh HJ, Reed EF, Lonnqvist J, Peltonen L, Woodward JA, et al. HLA-B maternal-fetal genotype matching increases risk of schizophrenia. Am J Hum Genet 2006; 79: 710-5.

11 Eaton WW, Byrne M, Ewald H, Mors O, Chen CY, Agerbo E, et al. Association of schizophrenia and autoimmune diseases: linkage of Danish national registers. Am J Psychiatry 2006; 163: 521-8.

12 Chang SH, Chiang SY, Chiu CC, Tsai CC, Tsai HH, Huang CY, et al. Expression of anti-cardiolipin antibodies and inflammatory associated factors in patients with schizophrenia. Psychiatry Res 2011; 187: 341-6.

13 Chen $\mathrm{CY}$, Liu CY, Su WC, Huang SL, Lin KM. Factors associated with the diagnosis of neurodevelopmental disorders: a population-based longitudinal study. Pediatrics 2007; 119: e435-43.

14 World Health Organization. International Classification of Diseases, Ninth Revision, Clinical Modification (ICD-9-CM). WHO, 1979

15 Chien IC, Hsu JH, Lin CH, Bih SH, Chou YJ, Chou P. Prevalence of diabetes in patients with schizophrenia in Taiwan: a population-based National Health Insurance study. Schizophr Res 2009; 111: 17-22.

16 Chen $\mathrm{CY}$, Liu CY, Su WC, Huang SL, Lin KM. Urbanicity-related variation in help-seeking and services utilization among preschool-age children with autism in Taiwan. J Autism Dev Disord 2008; 38: 489-97.

17 Benjamini Y, Drai D, Elmer G, Kafkafi N, Golani I. Controlling the false discovery rate in behavior genetics research. Behav Brain Res 2001; 125 279-84

18 Messias EL, Chen CY, Eaton WW. Epidemiology of schizophrenia: review of findings and myths. Psychiatr Clin North Am 2007; 30: 323-38.

19 Youinou P, Pers JO, Gershwin ME, Shoenfeld Y. Geo-epidemiology and autoimmunity. J Autoimmun 2010; 34: J163-7.

20 Manji N, Carr-Smith JD, Boelaert K, Allahabadia A, Armitage M, Chatterjee VK, et al. Influences of age, gender, smoking, and family history on autoimmune thyroid disease phenotype. J Clin Endocrinol Metab 2006; 91 4873-80.

21 Weetman AP. Graves' disease. N Engl J Med 2000; 343: 1236-48.

22 Othman SS, Abdul Kadir K, Hassan J, Hong GK, Singh BB, Raman N. High prevalence of thyroid function test abnormalities in chronic schizophrenia. Aust N Z J Psychiatry 1994; 28: 620-4.

23 Wright $\mathrm{P}$, Sham $\mathrm{PC}$, Gilvarry $\mathrm{CM}$, Jones $\mathrm{PB}$, Cannon $\mathrm{M}$, Sharma $\mathrm{T}$, et al. Autoimmune diseases in the pedigrees of schizophrenic and contro subjects. Schizophr Res 1996; 20: 261-7.

24 Eaton WW, Hayward C, Ram R. Schizophrenia and rheumatoid arthritis: a review. Schizophr Res 1992; 6: 181-92.

25 Mors O, Mortensen PB, Ewald H. A population-based register study of the association between schizophrenia and rheumatoid arthritis. Schizophr Res 1999; 40: 67-74.

26 Oken RJ, Schulzer M. At issue: schizophrenia and rheumatoid arthritis: the negative association revisited. Schizophr Bull 1999; 25: 625-38.

27 Torrey $\mathrm{EF}$, Yolken $\mathrm{RH}$. The schizophrenia-rheumatoid arthritis connection: infectious, immune, or both? Brain Behav Immun 2001; 15: 401-10.

28 Scott DL, Wolfe F, Huizinga TW. Rheumatoid arthritis. Lancet 2010; 376: 1094-108.

29 Shapira Y, Agmon-Levin N, Shoenfeld Y. Defining and analyzing geoepidemiology and human autoimmunity. J Autoimmun 2010; 34: J168-77.

30 Zeng QY, Chen R, Darmawan J, Xiao ZY, Chen SB, Wigley R, et al. Rheumatic diseases in China. Arthritis Res Ther 2008; 10: R17.

31 Raychaudhuri SP, Farber EM. The prevalence of psoriasis in the world J Eur Acad Dermatol Venereol 2001; 15: 16-7.

32 Chan JC, Liu HS, Kho BC, Lau TK, Li VL, Chan FH, et al. Longitudinal study of Chinese patients with pernicious anaemia. Postgrad Med J 2008; 84: 644-50.

33 Cummins AG, Roberts-Thomson IC. Prevalence of celiac disease in the Asia-Pacific region. J Gastroenterol Hepatol 2009; 24: 1347-51.

34 Watts RA. Epidemiology of vasculitis in India - what is known? J Indian Rheumatol Assoc 2005; 13: 8-15. 
35 Jia P, Wang L, Meltzer HY, Zhao Z. Common variants conferring risk of schizophrenia: a pathway analysis of GWAS data. Schizophr Res 2010; 122: 38-42.

36 O'Dushlaine C, Kenny E, Heron E, Donohoe G, Gill M, Morris D, et al. Molecular pathways involved in neuronal cell adhesion and membrane scaffolding contribute to schizophrenia and bipolar disorder susceptibility. Mol Psychiatry 2011; 16: 286-92.

37 Gregersen PK, Olsson LM. Recent advances in the genetics of autoimmune disease. Annu Rev Immunol 2009; 27: 363-91.
38 Shi J, Levinson DF, Duan J, Sanders AR, Zheng Y, Pe'er I, et al. Common variants on chromosome 6 p22.1 are associated with schizophrenia. Nature 2009: 460: 753-7.

39 Zenewicz LA, Abraham C, Flavell RA, Cho JH. Unraveling the genetics of autoimmunity. Cell 2010; 140: 791-7.

40 Trowsdale J. HLA genomics in the third millennium. Curr Opin Immunol 2005; 17: 498-504.

\section{Mrs Nicely Nice}

\section{Kate Evans}

Everything is always really

Nicely nice, in her life,

Lived on the edge of breakdown.

Not for her the cut and thrust of therapy,

Stripping the soul bare,

Re-building on rock.

Not for her work and rest,

The measured pace of days and nights,

The brilliant changes of the seasons. No.

Hers is a constant ferreting

Along the edge of the unendurable

Like a terrified fieldmouse,

The contagion of panic in her breast,

Running here, there,

Attempting to staunch the seepage of agony,

Stem each new treacherous leak,

Sandbag the river of her past against flood -

That past whose existence she constantly denies,

As it devastates her days, her weeks, her years. No, no,

She is not disturbed,

There is nothing wrong, of course

She's fine - can't you see?

That everything, really,

Is so nicely nice.

Kate Evans's poems are from Journey into Healing, published by Survivors' Poetry in 2006 as part of Survivors' Poetry Mentoring Pamphlet Series. She was mentored by Dave Russell. Another of her poems, On Magritte's Healing, will be published in a subsequent issue of the Journal.

Chosen by Femi Oyebode. 